\title{
Service users' experiences and views of aggressive situations in mental health care: a systematic review and thematic synthesis of qualitative studies
}

This article was published in the following Dove Press journal:

Journal of Multidisciplinary Healthcare

3 October 2015

Number of times this article has been viewed

\section{Camilla Buch Gudde ${ }^{1,2}$ \\ Turid Møller Ols $\varnothing^{4}$ \\ Richard Whittington ${ }^{1,5,6}$ \\ Solfrid Vatne ${ }^{3}$}

'Forensic Department Brøset, Centre for Research and Education in Forensic Psychiatry, St Olavs Hospital, ${ }^{2}$ Department of Social Work and Health Science, Faculty of Social Science and Technology Management, NTNU, Trondheim, ${ }^{3}$ Institute of Health and Social Sciences, Molde University College, Molde, ${ }^{4}$ Norwegian Resource Centre for Community Mental Health, NTNU Social Research AS, ${ }^{5}$ Department of Neuroscience, Norwegian University of Science and Technology, NTNU, Trondheim, Norway; ${ }^{6}$ Institute of Psychology, Health and Society, University of Liverpool, Liverpool, UK
Correspondence: Camilla Buch Gudde Forensic Department Brøset, Centre for Research and Education in Forensic Psychiatry, St Olavs University Hospital, PO Box 1803 Lade, 7440 Trondheim, Norway

Email camilla.gudde@ntnu.no
Background: Aggressive situations occurring within mental health services can harm service users, staff, and the therapeutic environment. There is a consensus that the aggression phenomenon is multidimensional, but the picture is still unclear concerning the complex interplay of causal variables and their respective impact. To date, only a small number of empirical studies include users' views of relevant factors. The main objective of this review is to identify and synthesize evidence relating to service users' experiences and views of aggressive situations in mental health settings.

Methods: We included qualitative studies of any design reporting on service users' own experiences of conditions contributing to aggressive situations in mental health care and their views on preventative strategies. Eligible articles were identified through an electronic database search (PsycINFO, PubMed, Ovid Nursing Database, Embase, and CINAHL), hand search, and cross-referencing. Extracted data were combined and interpreted using aspects of thematic synthesis.

Results: We reviewed 5,566 records and included 13 studies (ten qualitative and three mixed methods). Service users recognized that both their own mental state and negative aspects of the treatment environment affected the development of aggressive situations. Themes were derived from experiential knowledge and included calls to be involved in questions regarding how to define aggression and relevant triggers, and how to prevent aggressive encounters effectively. The findings suggest that incidents are triggered when users experience staff behavior as custodial rather than caring and when they feel ignored.

Conclusion: The findings highlight the importance of staffs' knowledge and skills in communication for developing relationships based on sensitivity, respect, and collaboration with service users in order to prevent aggressive situations. An important factor is a treatment environment with opportunities for meaningful activities and a preponderance of trained staff who work continuously on the development of conditions and skills for collaborative interaction with users.

Keywords: aggression, violence, service user experiences, inpatient, mental health, user involvement

\section{Background}

There has been an extensive research effort focused on aggressive situations within mental health services over the last 20-30 years. The great interest in the topic is probably linked to the fact that the consequences for those involved are significant. Aggressive and violent episodes can harm users and staff, damage the relationship and alliance between users and staff, and constitute a threat to the therapeutic environment on the wards. ${ }^{1,2}$ Aggressive situations can be understood as an action with intent to harm somebody, either in the absence or presence of a perceived threat, as in self-defense. 
The majority of studies in this area have focused on etiology and individualized explanations of aggression, ${ }^{3,4}$ how aggression is manifested, the characteristics of perpetrators, the injuries sustained, and the management of aggression. ${ }^{5}$ The most frequent focus has been on examining mentally ill people as perpetrators of violence and on staff safety issues. ${ }^{6-9}$ However, violence and aggressive behavior in any setting is a complex phenomenon with a variety of triggering factors, behaviors, and consequences beyond the individual perpetrator. A number of theories have been developed to explain the causes of the problem in mental health settings. Nijman's model includes an interaction between ward variables and staff variables leading to the emergence of an aggressive incident. ${ }^{10,11}$ Others have examined variations in how individual staff emphasize different domains when explaining violence, using internal, external, and situational/relational models. ${ }^{6}$ Existing models of patient aggression in inpatient settings are tentative according to Winstanley ${ }^{12}$ and lack both firm theoretical foundations and empirical support. Furthermore, no models are currently available which explain aggressive incidents from the patient's perspective. Nevertheless, a recent review ${ }^{13}$ of the limited evidence that is available concluded that there seems to be a disagreement between patients and staff concerning the predictors of aggressive episodes. Patients tend to emphasize to a greater extent the significance of environmental conditions and poor communication, while staff tends to rely on internal variables like the patient's illness as the main reason. Theoretically, there have been calls for a shift in perspective. Hamrin et $\mathrm{al}^{2}$ identified a complex interaction between patients, staff, and ward culture, and introduced a virtue ethic perspective moving away from a focus on rules and principles to refocus on relational competence within a culture characterized by relational ethics. Cutcliffe and Riahi ${ }^{14,15}$ proposed a systemic perspective, and argued for a more comprehensive understanding and conceptualization of aggression in inpatient mental health settings. Their systemic model focuses on the multidimensionality and complexity of aggression and proposes four broad thematic categories related to the client, the environment, the health care system, and the clinician. They argue for the need to broaden our knowledge related to the causes of aggression and violence, in order to gain a better systemic understanding of the phenomenon. There seems to be an agreement that the aggression phenomenon is multidimensional and multifactorial, but the picture is still unclear concerning how the complex interplay of variables operates and their respective impact. Alongside the overemphasis on individual patient factors, especially psychopathology, in theories and models of aggression in mental health settings, there is a real lack of user involvement in identifying relevant factors. These two issues go hand-inhand. Staff and professional researchers will tend to see the world in a particular way, and thus are likely to be more or less ignorant of how service users experience the world. When explanations of staff and patients for aggression are directly compared, they differ significantly in certain ways. ${ }^{6}$ Models of aggression must draw on the experience of both staff and service users if they are going to be comprehensive and form the basis of effective interventions. This will be in line with international guidance, which recommends the involvement of users and the inclusion of the users' perspective and knowledge in research and treatment. ${ }^{16,17}$ Despite a relatively sparse body of research literature concerning the users' experiences of violence and aggression, our initial scoping searches indicated that sufficient studies have been undertaken to warrant a systematic review of the findings. This paper reports a formal review of qualitative studies focusing on this perspective and provides a synthesis of the findings from the individual studies. In the following, the terms "patient" and "service user" will be used interchangeably.

\section{Research question}

The research question that guided the review was the following: What is known about service users' experiences of aggressive situations in mental health care settings? In particular, what are the service users' experiences of and views on 1) conditions contributing to aggressive situations in mental health care and 2) effective preventative strategies in these situations?

\section{Methods}

Methods for systematic reviewing of qualitative research are still emerging, and no consensus exists yet regarding its correct execution. ${ }^{18}$ Critics claim that qualitative research is epistemologically specific to a particular context, time, and group of participants, and therefore is unsuitable to be decontextualized through extraction and synthesis in a systematic review. ${ }^{19}$ However, excluding qualitative research in this way from formal reviews prevents its inclusion in evidence-based policy and practice development. This is highly undesirable, and so there is a growing interest in how to synthesize this type of research without violating its basic principles. ${ }^{20}$ One approach which attempts this is thematic synthesis, ${ }^{18}$ and we applied a modified version of this method to conduct a synthesis of qualitative studies relevant to the review questions. 


\section{Searching}

A comprehensive search strategy was developed to identify relevant studies and was applied to the following databases: MEDLINE/PubMed, PsycINFO, Embase, CINAHL, and Ovid Nursing Database for the period from their inception to October 2014. We used indexed terms and subject headings as well as free-text-word searches across four key concepts related to the review question, combined in various ways according to the requirements of each database. The following keywords and their synonyms were used with truncated wildcards where appropriate: "Service user" ("Patient", "mentally ill”, "mental disorder", "service user") AND "Experience" ("Perspective", "Experience", "View", "Attitude", "Opinion”) AND "Aggression" ("Violence", "Aggressive behaviour", "Assault", "Physical aggression", "Verbal aggression", "Threat", "Difficult behaviour") AND "Mental health service" ("Mental hospital", "Psychiatric hospital", "Psychiatric ward", "Psychiatric unit", "Mental health care", "Mental health service"). Papers were selected for full-text review by one researcher (CBG) based on scrutiny of the title or title and abstract against the inclusion criteria. Three researchers (CBG, TMO, and SV) then independently screened the full text of eligible papers $(n=65)$ based on the inclusion criteria. Disagreements on inclusion were resolved though discussion, and where necessary, arbitration by the third reviewer. In addition to the electronic search, reference lists of relevant articles and books produced by the search were screened.

\section{Inclusion and exclusion criteria}

Our inclusion criteria were based on the aim of the review rather than on a specific qualitative methodology or study design. ${ }^{21}$ All studies with an aim to qualitatively explore and/or report on service users' experiences or views of aggressive situations in mental health care settings were eligible. We included qualitative parts of mixed method studies, and also studies using structured questionnaires if these allowed the respondents to relate their experiences in an open-ended way. To be included, a study had to be qualitative and 1) have a population consisting of adult service users, 2) have a main focus on aggressive situations, 3) provide original qualitative data and 4) in English in a peer-reviewed journal. Articles were excluded if the topic was a minor part of the study focus or if they reported solely on the management rather than prevention of aggressive situations, that is, use of coercion.

\section{Quality assessment}

Evaluating the quality of primary research studies in a systematic review is complex and controversial as there is no accepted gold standard approach. ${ }^{20,22,23}$ Nevertheless, we wanted to evaluate the quality of each study in order to avoid drawing unreliable conclusions. ${ }^{18}$ Each included study was assessed by using the Critical Appraisal Skills Programme (CASP) checklist for purely qualitative studies, or where appropriate, the Mixed Methods Appraisal Tool (MMAT). ${ }^{24,25}$ Two researchers (TMO and CBG) independently assessed the quality of the included studies using one of these checklists; any disagreement between the reviewers was discussed until consensus was reached.

\section{Data extraction}

Decisions on what constitutes data for analysis are also less straightforward in qualitative reviews. Thomas and Harden ${ }^{18}$ extracted the entire text of the findings section in each included study (but nothing else) and subjected this text to thematic analysis. However, qualitative reports are complicated because they do not always obviously separate the study findings from the researchers' interpretation of the findings. ${ }^{18,19} \mathrm{We}$ firstly therefore closely read the whole paper to get an overall impression of it and to make sure that we did not miss relevant data written elsewhere than in the results or findings section of the paper. We then conducted a preliminary extraction of data based on the main review question: service users' experiences, which directed us to focus on any text in the entire paper, which reported basic study characteristics, aspects of the method, or the concept of service user's experiences, or views on aggressive situations. Two researchers (CBG and TMO) extracted this data from the included studies. Finally, we extracted all sections referring to the patients' views or experiences, either in the form of quotations from the patients themselves or in the form of the author commenting upon patients' views or experiences with or without reference to quotations (ie, patients freely used the word "punishment"). Extracted text was pasted into a self-made matrix for analysis and synthesis.

\section{Data synthesis}

The studies were analyzed and synthesized using Thomas and Harden's thematic synthesis approach ${ }^{18}$ with some modifications. The first step in the original approach involves free line-by-line coding, but our first step involved free coding of meaning units, defined as a text fragment containing any information about the research question. This could be a line, but it could also be whole sentences. Our second and third steps followed Thomas and Harden's approach specifically, that is, organization of free codes into related areas to construct descriptive themes which largely reflect the findings of the original studies, and then the development of analytical themes. To answer the sub-questions, 
we developed codes about the users' opinions of factors that contribute to development of aggressive situations, that is, strong and hierarchical rules and various examples of not being met from the staff with respect, and what could be effective preventive strategies in these situations, that is, being met with real dialogues, respect, and sensitivity.

These analytical themes aimed to go beyond the primary studies by generating new interpretive constructs capturing service users' experiences and understanding of aggressive situations in general.

\section{Results}

\section{Selection of studies}

Figure 1 outlines the search and selection strategy adopted.

Details regarding MMAT and CASP assessment criteria and scores for each study are presented in Table 1.

\section{Study demographics}

A total of 13 publications were included in the review which had been conducted in five countries: $\operatorname{UK}(n=6), \operatorname{USA}(n=2)$, Australia ( $n=2)$, Sweden $(n=2)$, and Canada $(n=1)$. The studies were published between 1995 and 2007, with just over half published between 2003 and $2006(n=8)$. They were predominantly purely qualitative in design $(n=10)$, although three used mixed methods. One study reported on qualitative data gathered in the course of a 3-year ethnographic study, and accordingly, it was not possible to identify the sample size. ${ }^{26}$ Information about the nature of the mental health service and definitions of the type of psychiatric ward varied across the studies, but both forensic and non-forensic psychiatric settings were included. Nine of the studies reported only from non-forensic settings, ${ }^{6,26-33}$ two studies were from medium-secure/high-secure forensic settings, ${ }^{7,9}$ and one had respondents from both forensic and non-forensic settings. ${ }^{34}$ In one study, the patients' experiences were not related to a specific ward or unit but examined general experiences from an earlier hospitalization. ${ }^{8}$ Details of study characteristics, aim, design, methods, and key findings are provided in Table 2.

\section{Thematic analysis of service users' experiences and views of aggressive situations}

Patients had a broad definition of what constituted aggressive and violent incidents including both conflicts with other patients ${ }^{7-9,26}$ and staff aggression toward patients. ${ }^{8}$ In terms of the users' experiences and views of conditions that contributed to aggression factors that might help to prevent the development of aggressive incidents, five descriptive

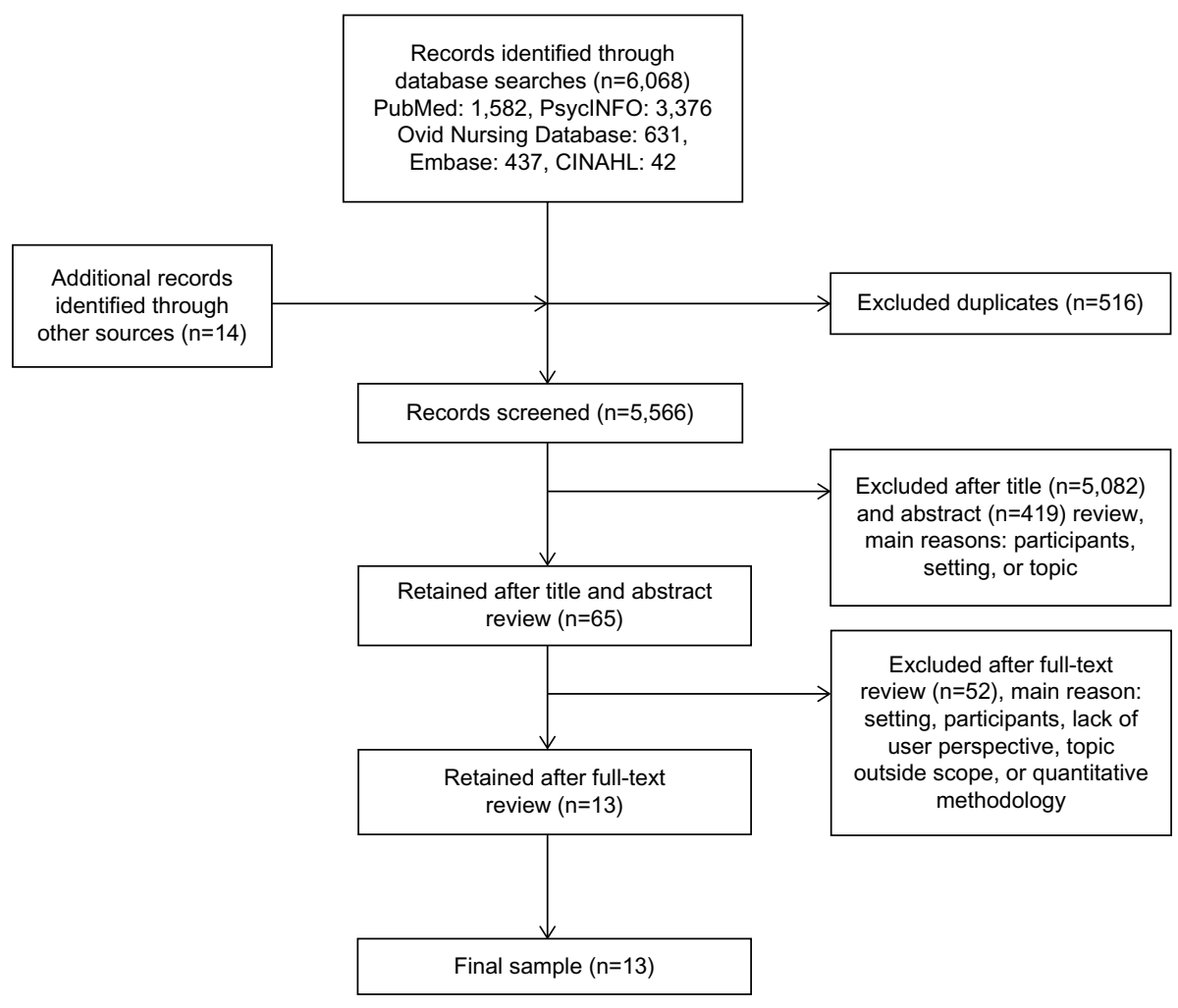

Figure I Search and selection strategy. 
Table I Quality assessment of included studies

\begin{tabular}{|c|c|c|c|c|c|c|c|c|c|c|}
\hline \multirow[t]{2}{*}{ Qualitative studies } & \multicolumn{10}{|c|}{$\begin{array}{l}\text { CASP qualitative quality criteria } \\
\text { met }^{\mathrm{a}}\end{array}$} \\
\hline & A & B & C & D & $\mathbf{E}$ & $\mathbf{F}$ & G & $\mathbf{H}$ & $\mathbf{I}$ & $J$ \\
\hline Bensley et al $(1995)^{27}$ & $\checkmark$ & $x$ & $x$ & $\checkmark$ & $x$ & $\checkmark$ & $x$ & $x$ & $\checkmark$ & $\checkmark$ \\
\hline Benson et al $(2003)^{28}$ & $\checkmark$ & $\checkmark$ & $\checkmark$ & $x$ & $\checkmark$ & $x$ & $\checkmark$ & $\checkmark$ & $\checkmark$ & 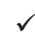 \\
\hline Bonner et al $(2002)^{29}$ & $\checkmark$ & $\checkmark$ & $\checkmark$ & $\checkmark$ & $\checkmark$ & $\checkmark$ & $\checkmark$ & $\checkmark$ & $\checkmark$ & 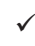 \\
\hline Carlsson et al (2006) ${ }^{34}$ & $\checkmark$ & $\checkmark$ & $\checkmark$ & $x$ & $\checkmark$ & $x$ & $\checkmark$ & $\checkmark$ & $\checkmark$ & $\checkmark$ \\
\hline Hinsby and Baker (2004) & $\checkmark$ & $\checkmark$ & $\checkmark$ & $x$ & $\checkmark$ & $x$ & $\checkmark$ & $\checkmark$ & $\checkmark$ & \\
\hline Johnson et al $(1997)^{31}$ & $\checkmark$ & $\checkmark$ & $\checkmark$ & $x$ & $\checkmark$ & $\checkmark$ & $x$ & $\checkmark$ & $\checkmark$ & 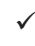 \\
\hline Johnson and Delaney $(2007)^{32}$ & $\checkmark$ & $\checkmark$ & $\checkmark$ & $\checkmark$ & $\checkmark$ & $\checkmark$ & $\checkmark$ & $x$ & $\checkmark$ & \\
\hline Kumar et al $(200 \mathrm{I})^{8}$ & $\checkmark$ & $\checkmark$ & $\checkmark$ & $\checkmark$ & $\checkmark$ & $x$ & $\checkmark$ & $\checkmark$ & $\checkmark$ & 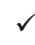 \\
\hline Meehan et al (2006) ${ }^{9}$ & $\checkmark$ & $\checkmark$ & $\checkmark$ & $\checkmark$ & $\checkmark$ & $\checkmark$ & $\checkmark$ & $\checkmark$ & $\checkmark$ & \\
\hline Quirk et al $(2004)^{26}$ & $\checkmark$ & $\checkmark$ & $\checkmark$ & $\checkmark$ & $\checkmark$ & $\checkmark$ & $x$ & $x$ & $\checkmark$ & 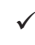 \\
\hline \multirow[t]{2}{*}{ Mixed method studies } & \multicolumn{10}{|c|}{ MMAT quality criteria met ${ }^{\mathrm{b}}$} \\
\hline & $\mathbf{I}$ & & & 2 & & & 3 & & & 4 \\
\hline Duxbury and Whittington (2005) & $\checkmark$ & & & $\checkmark$ & & & $\checkmark$ & & & 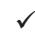 \\
\hline Illkiw-Lavalle and Grenyer $(2003)^{30}$ & $\checkmark$ & & & $\checkmark$ & & & $\checkmark$ & & & \\
\hline Omerov et al $(2004)^{33}$ & $\checkmark$ & & & $\checkmark$ & & & $\checkmark$ & & & $x$ \\
\hline
\end{tabular}

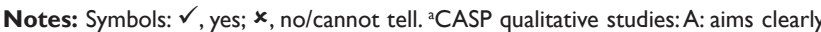
stated; B: appropriate methodology; C: appropriate design to address the aims of the research; D: appropriate recruitment strategy; E: data collected in a way that addressed the research issue; $\mathrm{F}$ : adequate consideration of relationship between researcher and participants; G: consideration of ethical issues; H: sufficiently rigorousness in data analysis; I: findings clearly stated; J: consideration of relevance and transferability of the research; 'MMAT (mixed method studies): I: relevant design; 2: relevant integration of data; 3 : appropriate considerations; 4 : appropriate criteria for qualitative/quantitative components.

Abbreviations: CASP, Critical Appraisal Skills Programme; MMAT, Mixed Methods Appraisal Tool.

themes were identified: i) themselves as unstable and lacking control, ii) being behind locked doors - isolated and frightened, iii) being in need of protection and stimulation through meaningful activities, iv) feeling powerless and ignored, and v) in need of a caring relationship with trained staff. Further development then yielded three analytical themes which are discussed below: i) being in an unstable mental condition - self-protective strategies, ii) experiencing the ward as custody rather than care, and iii) user involvement to prevent violence based on early intervention and real dialogue. The findings are structured below according to these analytic themes, along with the descriptive themes. The themes overlapped with each other somewhat in an ongoing circular process.

\section{Being in an unstable mental condition - self-protective strategies}

Themselves as unstable and in lack of control

Some articles presented findings that showed patients' awareness of how their mental symptoms were one of the conditions that was important in making them feel upset prior to the aggressive incident. The patients referred to being "in the acute stage", being manic or desperate, and hearing voices or experiencing delusions. ${ }^{29,30,32,33}$ Other emotions frequently described as precursors were frustration, irritability, anger, and feeling unsafe or anxious. ${ }^{28,30,32,34} \mathrm{In}$ some studies, these feelings related to reactivation of earlier negative experiences. ${ }^{28,32}$ In several studies, patients talked about being unstable and feeling a sense of being out of control in themselves and that it took little to get them out of balance. ${ }^{7,929,31}$ Some patients experienced a sense of being in control, but only up to a certain point, and some expressed the view that they felt able to control the degree or intensity of their aggressive behavior to a certain extent. ${ }^{9,31}$ Further, some patients felt that demands from the staff for them to control their emotions in order to maintain peace and structure on the ward were unrealistic and unachievable. ${ }^{7}$ Useful strategies to keep emotions under control were to actively seek out staff for support or to use a combination of diverting strategies, for example, reading, drawing, or physical activity, avoiding difficult situations, or withdrawal. ${ }^{31,32}$ Patients highlighted how they had given the staff warnings about potential aggression or showed clear signals of distress over a long period without receiving any interventions, leaving them with feelings of not being taken seriously, ignored, or being misinterpreted as uncooperative. ${ }^{8,29,33,34}$

It is not actual violence but, ... the precursor, the thing that leads up to it ... the things that can go on for weeks and weeks ... has nothing to do with aggression ... are just pushed aside. $^{8}$

\section{Experiencing the ward as custody rather than care}

Patients' feelings of being out of control were frequently made worse by an environment characterized by unpredictability, insecurity, and oppressiveness, and individual protective strategies were difficult to use for many or were ineffective in an environment characterized by closed doors, lack of available staff, and general turbulence on the ward.

In most of the included studies, the patients emphasized characteristics of the ward environment as factors that might contribute to the development of aggressive events. ${ }^{6-9,26,27,29-31,33,34}$ The atmosphere created by the negative ward structures sometimes felt custodial rather than caring.

\section{Behind locked doors - feeling isolated and frightened}

The ward environment was frequently described negatively, especially in terms of being busy, turbulent, overcrowded, and noisy with a stressful and unpredictable atmosphere. Participants in several studies perceived the ward as an 


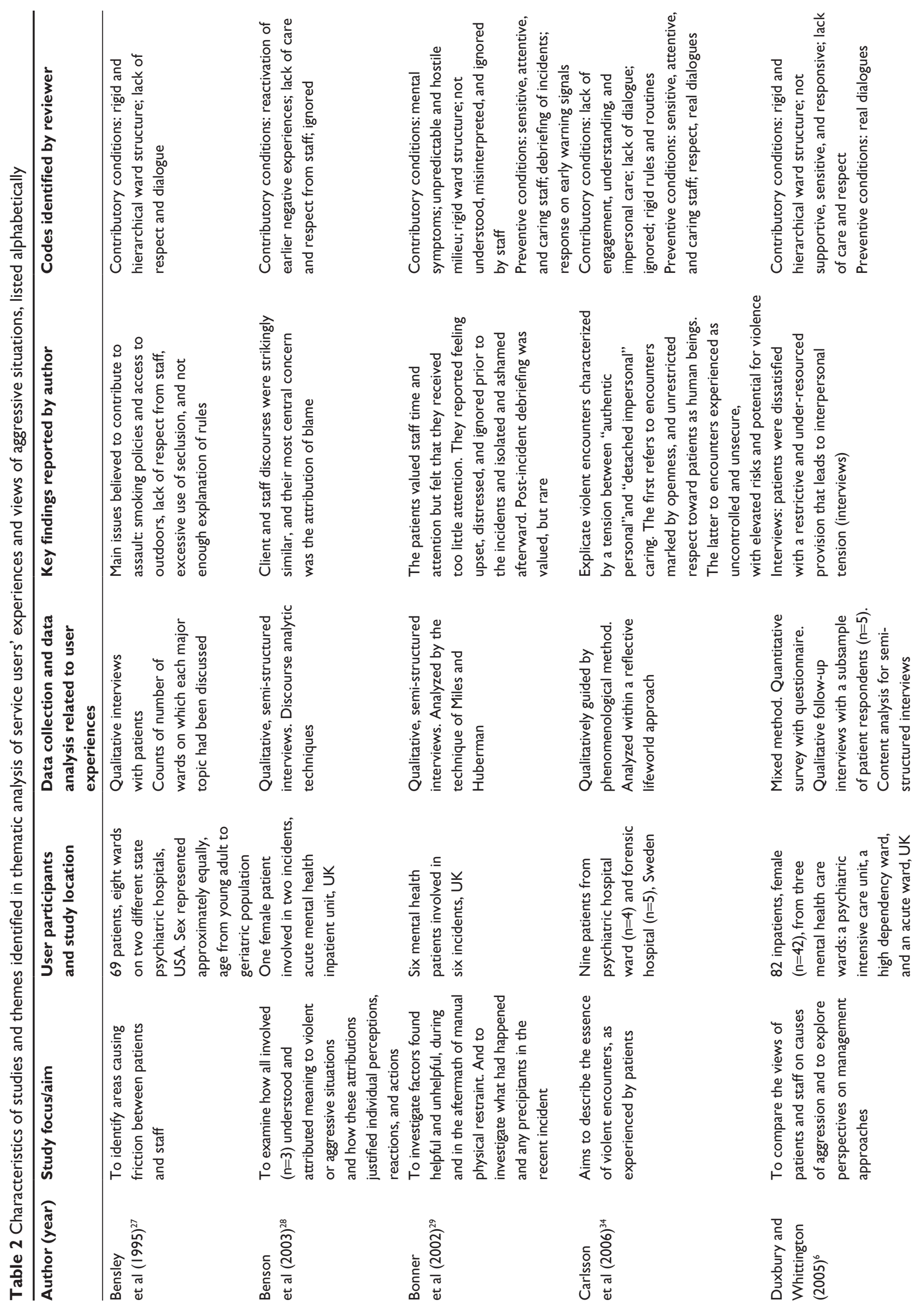



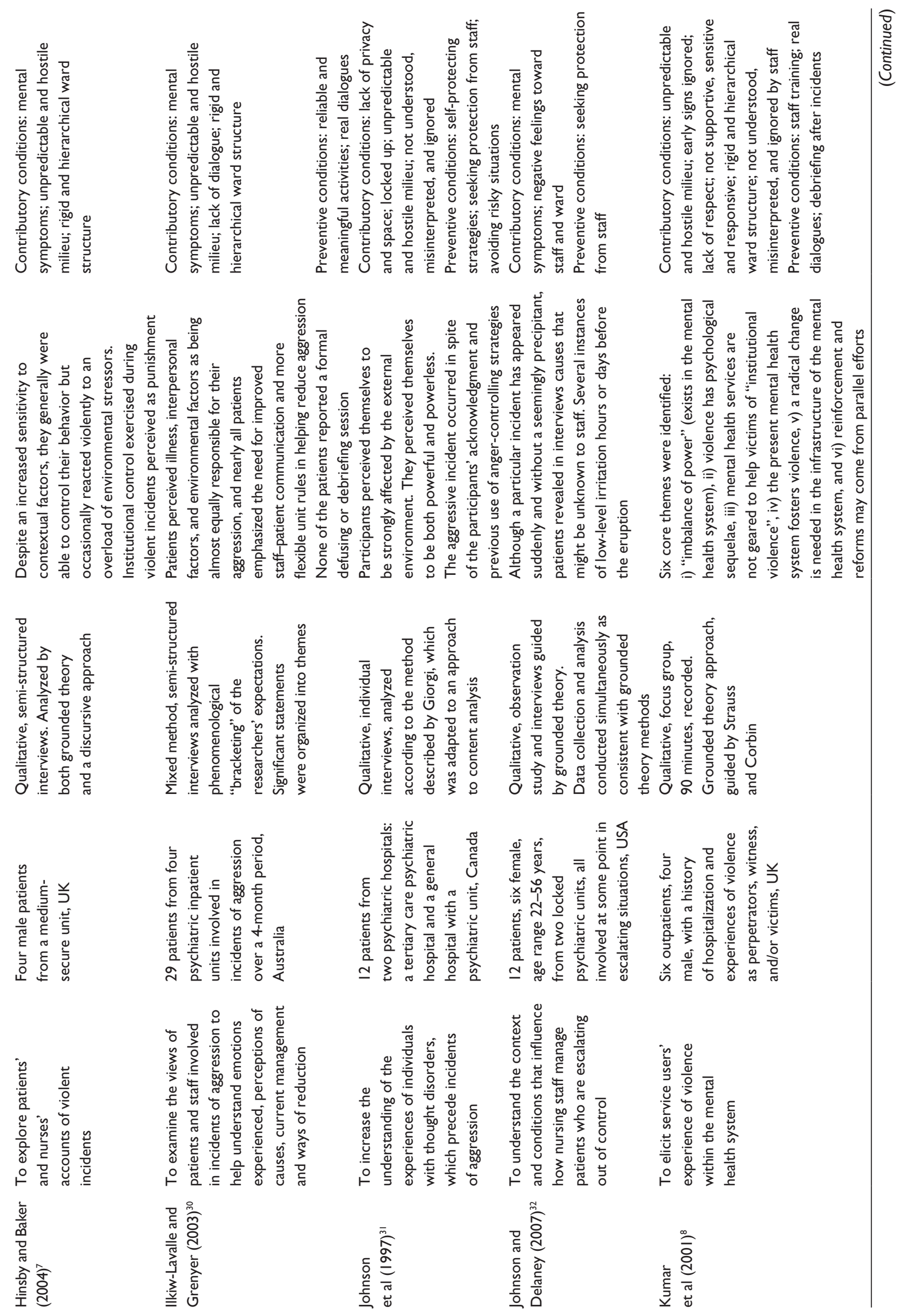


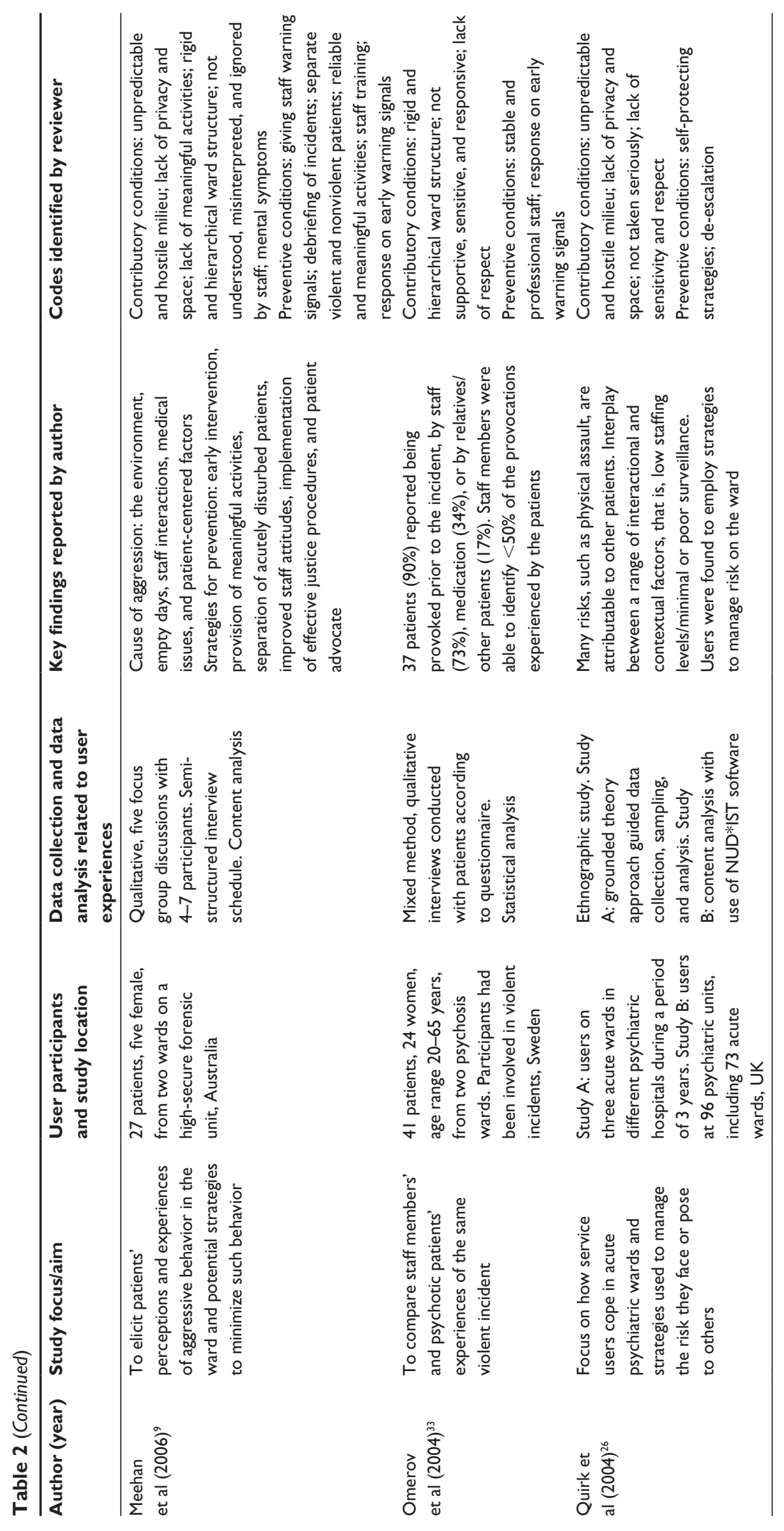


unsafe and frightening place causing feelings of anxiety for them. ${ }^{9,26,27,29}$ This was alongside other factors, such as a concentration of numerous patients with different kinds of behavioral difficulties in one place, ${ }^{8,9,26,29,31}$ which left few opportunities to develop social relationships. ${ }^{26}$ Frequent provocations and disagreements between patients were mentioned, particularly regarding money, smoking, and music and TV choices. Some users described an unpredictable and hostile environment characterized by intimidation, bullying, lack of communication, and misapprehension between users who did not like each other. ${ }^{26,27,30,31}$ It was perceived to be particularly difficult when patients with a drug problem were resident on the ward and particularly insecure when fellow patients took control of the ward without intervention from staff. ${ }^{26}$ Furthermore, patients encountered difficult and sometimes dangerous and impulsive fellow patients who caused tension among patients and between patients and staff. ${ }^{9}$ Restricted permission to leave in combination with overcrowding and lack of privacy and space on the ward was highlighted as significant, in addition to limited opportunities to withdraw in order to get a break from all that took place on the ward. 9,26,27,30,31 The hierarchical structure that characterized the ward was another factor that contributed to these aggressive incidents. ${ }^{6,27,30,31,34}$ The system was perceived as ambiguous with elements of both care and control, and the environment was described as over-reliant on coercion with a major focus on safety and control. ${ }^{7}$ Petty or unreasonable restrictions placed upon the patients were frequently mentioned, especially denial of minor everyday requests, for example, use of a telephone, cigarettes, or a cup of coffee outside the scheduled times. Further, withdrawal of ward privileges and freedom, for no apparent reason, was also seen as an antecedent of aggressive behavior. ${ }^{9,29,31,33,34}$ Rules that were perceived as incomprehensible, for example, a common bedtime for all on the ward, ${ }^{7}$ and unfair were emphasized as triggering factors to aggression, especially if vaguely communicated. ${ }^{27,29}$ Some of the daily procedures, like standing in a queue outside the nurses' office waiting for medicine, were interpreted as humiliating. ${ }^{9}$ Respondents were concerned about rigidity, lack of flexibility, and the

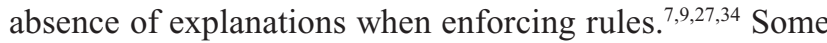
stated that it was not necessarily the rules themselves that were the problem, rather the way they were enforced and communicated to the patients.

They may be saying exactly the same thing as the other nurse, saying, "No, you can't do this, do that" but it is in the way it is said, and the reasons for it are given. ${ }^{7}$
Thus, the combination of being separated from general society, confined behind locked doors in a turbulent and frightening environment, and thus being unable to leave the hospital was perceived as a heavy burden. Some even mentioned self-discharge or absconding as alternatives to avoid risky situations. ${ }^{26}$

\section{In need of protection and stimulation through meaningful activities}

One study reported in detail patients' strategies to reduce the sense of being at risk from fellow patients. ${ }^{26}$ Strategies mentioned were to seek protection from staff, particularly those who they knew well and who had taken the patient's experiences and worry seriously in the past. Further, strategies involved avoidance and withdrawal from risky situations and threatening patients, and identification of safe places for withdrawal on the ward. The TV room was preferred to the smoking area for withdrawal, as the latter was perceived as an unsafe place with a lot of friction, especially when the staff was absent.

The experience of being on the ward was in some of the studies characterized by a feeling of boredom and enforced idleness. Lack of stimulation and meaningful activities was mentioned as a source of frustration and aggression, and respondents in the studies were concerned about opportunities for meaningful activities which would counteract boredom and inactivity., ${ }^{9,26,27}$ In one study, patients expressed frustration at being forced to wait for scheduled activities that in the end were canceled and expressed the opinion that a regular and reliable daily program of activities, including physical activity and access to outdoor facilities, would have prevented aggression. $^{9}$

I think boredom is the biggest problem in here. If you are not busy doing something, you are just sitting around smoking all the time, if you are doing something it is better, that would prevent anger I think. Something worthwhile ... maybe we need to dig up the garden or something like that. ${ }^{9}$

\section{Feeling powerless and ignored - in need of a caring relationship with a trained staff}

The way the staff acted toward the patients could either provoke or prevent the occurrence of aggressive situations. ${ }^{6,8,9,26-31,33,34}$ Experiences of not being understood, misinterpreted, and ignored by the staff were especially provoking. $8,9,29,31,34$ In two studies, the patients referred to staff as being absent and detached, sitting behind locked doors in their office or in a glass cage, while patients were left alone with restricted possibilities for communication and natural 
interaction. ${ }^{8,34}$ The perceived absence or avoidance of staff was experienced as representing a lack of understanding of the patients' distress at being left alone in stressful, turbulent, and insecure environments. Communication with nurses was frequently described as one-sided, contradictory, argumentative, hard, poor, or totally lacking, characterized by a lack of respect and insufficient understanding of the patient's problems. ${ }^{6,9,27,29,30,34}$

I got angry because they wouldn't listen to what I was trying to tell them. Telling them that I needed help, wanted to hurt myself ... it was horrible, I never want it to happen again. ${ }^{29}$

Patients experienced a lack of empathy and care from staff, ${ }^{6,9,28,34}$ who were felt to be unsupportive, insensitive, and unresponsive, and interactions were perceived to be disrespectful, unwarranted, and sometimes humiliating. $8,27,30,33,34$ Patients in two studies stated that staff did not show any genuine interest in the patients as human beings but simply restricted their role to being a professional doing their job. ${ }^{28,34}$ In one study, the patients experienced a feeling of ignorance and that led to a sense of inner violation with a feeling of not being worthy of the presence of the carer. The experience of violation directed to the patient as a human being could trigger a violent encounter. ${ }^{34}$ Such events might be perceived as a possibility to experience empowerment but was rooted in a feeling of powerlessness. ${ }^{31,33}$

\section{User involvement based on early intervention and real dialogue}

Patients had a need for real dialogue with staff, including the opportunity to express their feelings to carers who respected these feelings. Training and education of the staff was emphasized as fundamental to improving bad and patronizing attitudes among the staff. Important areas of staff training and education mentioned were defusing tension and performance of care rather than custody. ${ }^{8,9}$ Increased knowledge and ability among staff in order to distinguish verbal and nonverbal aggression and to allow normal reactions and to show feelings without overreaction were highlighted. ${ }^{8,34}$ In one of the studies, patients highlighted staff qualities like being engaged, understanding, and warm, and showing a sincere, straightforward, and unfeigned engagement, like the respect a carer shows toward another ordinary human being who is not a patient. ${ }^{34}$ In another study, patients preferred less reliance on agency nurses and other nonpermanent staff in order to increase the proportion of permanent staff and supported involving users in both training and education of staff as well as in staff recruitment. ${ }^{8}$ Respondents in the studies expressed a desire to talk about the incident, and to be asked what they felt and why they acted as they did. $8,9,29,34$ The sense of being violated could remain after an aggressive situation with patients experiencing strong emotions like being embarrassed, scared, and feeling empty and insignificant afterward. ${ }^{29,34}$

Further, patients called for greater use of early interventions, and more sensitivity and proactivity from staff, particularly when responding to problematic events and warning signs. Staff should be able to listen to and negotiate with the patients in order to calm down the situation. $8,9,30,34$ Furthermore, some wanted access to the formal reports submitted by staff about the aggressive event, and to have the opportunity to clear up any misunderstanding and/or give their own version of the incident. They emphasized the value of talking with aggressive patients and witnesses soon after the incident, in order to clarify exactly what happened and to contribute to avoiding future incidents., ${ }^{9}, 29$

Talk it through with them first to figure out exactly what has happened, you can't throw them into seclusion without first talking to them. I think it is good to have the staff to sort of calm the situation down first off ... but you have got to help those two that have been in that situation otherwise it will happen again. ${ }^{9}$

\section{Discussion}

Findings from this review illustrate that users recognize that the conditions that contribute to aggressive situations involve a combination of their own mental ill-health, an overload of negative structures, and a lack of positive structures in the treatment environment. Many described themselves as emotionally unstable at the time of an aggressive event while proactively struggling to protect themselves against provocations in the environment from both fellow patients and staff. They could not escape the negative situations being felt trapped behind locked doors, leading to a sense of being in custody rather than care. They felt that they tried to signal their distress and need for protection and support to staff but did not get adequate responses, leading to feelings of being ignored. These findings and relevant literature will be further discussed below around three central themes: 1) feeling ignored as a human being in custody rather than in care, 2) aggressive behavior as a kind of self-defending strategy, and 3) user involvement as a possible preventative strategy. 


\section{Feeling ignored as a human being in custody rather than in care}

It is well known from both the literature ${ }^{35}$ and practice that users with mental health problems can have an individual vulnerability based on congenital and/or environmental factors which becomes operative when exposed to certain current triggers or stressors in the environment. This knowledge is an important background for understanding the individual patients' vulnerability and sensitivity in specific situations and interactions. Psychiatric patients generally do not behave aggressively, but certain people are prone to aggressive behavior in specific situations and relationships. ${ }^{36}$ The findings from the current review are consistent with this view. A psychiatric ward can be a highly provoking and aversive place to be forced to inhabit, and mental illness is neither necessary nor sufficient to explain aggressive incidents that occur upon them. Users highlighted feeling trapped in an environment based on a strict hierarchy and rigid rules for behavior with an impoverished atmosphere lacking access to meaningful activities and supportive relationships with the staff. They often felt that the staff did not give them a sense of predictability or safety in this environment, when such predictability might help in preventing outbreaks of aggressive behavior. International literature also indicates that a good treatment milieu is best achieved with a stable and experienced staff group, clear leadership, clearly structured staff roles, and predictable, meaningful activities for patients. ${ }^{15}$ Recent studies also reemphasize the importance of being met with respect from the therapist as a central condition for therapeutic growth. The sensitivity of the therapist is as fundamentally important as the other core skills values and skills of trust, empathy, support, authenticity, and nonjudgmental feedback. ${ }^{34,37-40}$ When these conditions are missing, the users' experience tends to be one of custody rather than the care they were supposed to receive. It is also clear that feelings of powerlessness ${ }^{39}$ and being invaded ${ }^{39,41}$ are created when care systems exclusively aim to control patients, or where patients themselves feel deprived of control or the ability to act constructively by ward practices. Users' suggested strategies for aggression prevention in this review are also concentrated mainly around improvements in the environment and in the relationship between users and staff, with a particular plea that the staff act more proactively and intervene earlier before situations can escalate. Numerous studies have highlighted a connection between unmet patient requests, controlling staff behavior and aggressive incidents. ${ }^{39,42,43}$ This connection is common sense when explaining aggression in other settings but needs to be constantly reiterated when discussing aggression in the context of mental illness where the illness aspect tends to override any awareness of environmental triggers. A sense of limited autonomy stands out as an important factor at both a structural level, for example, locked doors and smoking restrictions, and at an individual or relational level, for example, a sense of inconsistency in the application of ward rules and the way that rules are communicated to the patients. ${ }^{2,17,43}$ The consequence of this is that patients do not have a sense of protection or support. On the contrary, they feel their care needs are ignored or not met in a respectful way, leading to an experience of violation of their own identity as a human being. The findings from the current review also reveal that patients in a vulnerable mental state, locked in an environment very different from their daily life, might end up feeling threatened, violated, and frightened at the very time when they are in need of care and support. When patients described how both their mental illness and the treatment environment they are in can induce anxiety, attempts at self-care through a struggle to gain control in interaction with other patients or staff, and thus, to protect self-esteem and self-respect, can be seen as a natural act.

\section{Aggression as self-defense}

One of the articles in the review by Carlsson et al ${ }^{34}$ reported that for the patients, the worst aspect of degrading treatment is the experience of inner violation as a human being with a feeling of being totally ignored and without dignity, and may lead to an urge for self-defense, sometimes in aggressive and violent ways. Coleman et $\mathrm{al}^{44}$ assert that the experience of violation is one of the strongest and most intense human emotions, rooted in earlier experiences of trauma, and created by social norms and rules in, for example, families and societies. The violation forms the framework of how we act in similar situations of powerlessness. ${ }^{44}$ This might also give support to Winstanley's cognitive model of patient aggression in which what staff perceive as aggression is intended by patients as an act of self-defense against perceived attack. According to this model, staff behavior is perceived as threatening rather than caring, and the consequent anxiety might evoke an aggressive response. ${ }^{12}$ The feeling of being ignored is very hard to live with and might trigger aggressive actions. Service user's aggressive behavior in this context therefore can be understood as a self-defense based on a feeling of being totally ignored. When one's identity as a human being is threatened, primal defensive actions and self-defense might 
be experienced as a form of empowerment in order to regain control over one's environment. Although this is not a positive strategy, it is comprehensible as a strategy to preserve the individual's self-esteem and self-respect in a situation of felt powerlessness. ${ }^{45}$

\section{Dynamic user involvement in preventing aggressive situations - a possible strategy?}

An important finding in this review is the users' views on the connection between their own unstable condition and aggressive situations, and their argument that user involvement in decision making might be preventive or enable more effective handling of such situations. Some users gave clear examples of how they tried to solve situations, giving signals and warnings to staff of potential problems, without these being recognized. They believed that preventive actions have to be based on good contact and dialogue with trained staff, which they know well, and can relate to and interact with confidently. Many studies also highlight the need for training and preparation in communication and interpersonal skills. ${ }^{39}$ Research also shows that user involvement is found to be difficult to practice in mental health, due to disability, illness, lack of user confidence, some staff resistance, and traditional professional roles. ${ }^{16}$ Oeye et $\mathrm{al}^{46}$ highlight the challenges including tensions between implementing individual user participation and maintenance of collective "house rules", and difficulties establishing equal relationships within the hierarchical hospital structure. The development of user involvement and patient autonomy seems to have been even slower in forensic settings because of the safety and security needs of patients, staff, and society. ${ }^{47}$ Users in the present review reported strong emotions in the aftermath of incidents, and again expressed a need for dialogue. They believed that user involvement both prior to and after an aggressive episode, for example, various types of debriefing or post-incident reviews, would probably have a preventive effect on future events. The process of debriefing functions as a way of both establishing agreed facts about what really happened and being a forum for provision of emotional support. ${ }^{48}$ According to Bonner and Wellmann, ${ }^{49}$ there is a growing research base indicating that aggressive incidents can lead to serious psychological impact on patients and staff, and thus, there is a clear need for post-incident support for both parties. Currently, there is little research or guidance concerning the recommended content and structure of such support. It remains important to ensure a structured process for all involved and to include users in framing the process, at individual, service, and system levels. One important approach to user involvement is an implementation of individualized violence risk management strategies that invite patients to contribute their knowledge concerning their own personal specific warning signs and interactional vulnerabilities. If patients have warning signs that are of an intrapsychological nature, for example, particular thoughts, these signs will be inaccessible to nurses' observations without behavioral expression. If user involvement and cooperation can be developed, important information can be added to treatment and risk management plans. ${ }^{1}$ The Early Recognition Method (ERM) aims to improve collaboration between nurses and patients to prevent aggression in forensic psychiatric care, and there is evidence that a focus on early signs of aggression identified in cooperation with patients led to a significant decrease in inpatient incidents. ${ }^{50}$ The ERM protocol helps to embed risk management into clinical practice and thereby supports staff and patients in working together to prevent aggression and violence. This again reiterates the need for a stronger focus on user involvement and patient participation in inpatient mental health settings to prevent aggressive incidents.

\section{Study limitations and strengths}

The present review is comprehensive and applies a rigorous methodology to summarize evidence in an important but complex area. It is limited by the relatively low quality of some of the included studies. Despite this limitation, we included the lower quality studies due to the small number of available papers on the topic and the lack of a clear consensus on defining quality in this type of research. The exclusion of studies not published in English may have contributed to a selection bias as services and issues will be different in nonEnglish-speaking countries and additional valuable ideas may have been gained from these alternative cultures. Some of the studies here did not discriminate in a clear way between the study results and the discussion; this may have caused some confounding of patients' descriptions and the authors' interpretation. It is never possible anyway to access the unmediated patient's voice in research texts, since even direct quotes are selected and framed by the study authors, but some attempt was made here to distinguish between "findings" and "interpretations". Furthermore, we extracted only those parts of the studies that covered the development and prevention of aggressive situations, thus avoiding sections where the patient might have reported satisfaction regarding support and safe treatment conditions. This might lead to a skewed picture in the review but is in accordance with the scope and aims set out for the study. The inclusion of both forensic and non-forensic 
wards, of patients' perspectives independent of diagnosis or severity of mental state, and (English-language) studies from different cultures and countries, might lead to difficulties in drawing robust contextual conclusions. However, by focusing on the phenomena of the patients' experiences and perception of aggressive situations in mental health settings, the review has provided some valuable insights.

\section{Conclusion}

From a patient perspective, this study highlights the importance of staffs' knowledge and skills in communication and collaboration with patients to prevent aggressive encounters. Therefore, a major ethical requirement and professional challenge is to develop relationships with patients based on sensitivity, respect, and collaboration. The main conclusion is the absolute need for services to provide a treatment environment with opportunities for meaningful activities and a preponderance of educated and trained staff who work continuously to improve collaborative interaction with the patients. International research indicates that staff might experience patients' behavior as both challenging and threatening. The intention and solution here is not to blame the staff but rather to reemphasize the importance of appropriate knowledge development and training in improving appreciative and therapeutic interaction with patients. The patients clearly articulated important insights gained from their experience and expressed a strong desire to be more involved in questions regarding how to define, understand, prevent, and manage aggressive situations effectively.

\section{Disclosure}

The authors report no conflicts of interest in this work. The views and opinions expressed herein are those of the authors and do not necessarily reflect those of the authors of the papers included in the review.

\section{References}

1. Eidhammer G, Fluttert FA, Bjørkly S. User involvement in structured violence risk management within forensic mental health facilities - a systematic literature review. J Clin Nurs. 2014;23(19-20):2716-2724.

2. Hamrin V, Iennaco J, Olsen D. A review of ecological factors affecting inpatient psychiatric unit violence: implications for relational and unit cultural improvements. Issues Ment Health Nurs. 2009; 30(4): 214-226.

3. Gadon L, Johnstone L, Cooke D. Situational variables and institutional violence: a systematic review of the literature. Clin Psychol Rev. 2006; 26(5):515-534.

4. Steinert T. Prediction of inpatient violence. Acta Psychiatr Scand. 2002; 106(s412):133-141.

5. Jansen GJ, Dassen TW, Groot Jebbink G. Staff attitudes towards aggression in health care: a review of the literature. J Psychiatr Ment Health Nurs. 2005;12(1):3-13.
6. Duxbury J, Whittington R. Causes and management of patient aggression and violence: staff and patient perspectives. J Adv Nurs. 2005;50(5):469-478.

7. Hinsby K, Baker M. Patient and nurse accounts of violent incidents in a medium secure unit. J Psychiatr Ment Health Nurs. 2004;11(3): 341-347.

8. Kumar S, Guite H, Thornicroft G. Service users' experience of violence within a mental health system: a study using grounded theory approach. J Ment Health. 2001;10(6):597-611.

9. Meehan T, McIntosh W, Bergen H. Aggressive behaviour in the highsecure forensic setting: the perceptions of patients. J Psychiatr Ment Health Nurs. 2006;13(1):19-25.

10. Nijman H. A model of aggression in psychiatric hospitals. Acta Psychiatr Scand. 2002;106(s412):142-143.

11. Nijman HL, áCampo JM, Ravelli DP, Merckelbach HL. A tentative model of aggression on inpatient psychiatric wards. Psychiatr Serv. 1999;50(6):832-834.

12. Winstanley S. Cognitive model of patient aggression towards health care staff: the patient's perspective. Work Stress. 2005;19(4):340-350.

13. Cornaggia CM, Beghi M, Pavone F, Barale F. Aggression in psychiatry wards: a systematic review. Psychiatry Res. 2011;189(1):10-20.

14. Cutcliffe JR, Riahi S. Systemic perspective of violence and aggression in mental health care: towards a more comprehensive understanding and conceptualization: part 1. Int J Ment Health Nurs. 2013;22(6): 558-567.

15. Cutcliffe JR, Riahi S. Systemic perspective of violence and aggression in mental health care: towards a more comprehensive understanding and conceptualization: part 2. Int J Ment Health Nurs. 2013;22(6): 568-578.

16. Storm M, Edwards A. Models of user involvement in the mental health context: intentions and implementation challenges. Psychiatr $Q$. 2013;84(3):313-327.

17. WHO. Improving Health Systems and Services for Mental Health. Geneva: WHO; 2009.

18. Thomas J, Harden A. Methods for the thematic synthesis of qualitative research in systematic reviews. BMC Med Res Methodol. 2008; $8(1): 45$.

19. Sandelowski M, Barroso J. Handbook for Synthesizing Qualitative Research. New York: Springer Publishing Company; 2007.

20. Harden A, Garcia J, Oliver S, et al. Applying systematic review methods to studies of people's views: an example from public health research. J Epidemiol Community Health. 2004;58(9):794-800.

21. Smith V, Begley CM, Clarke M, Devane D. Professionals' views of fetal monitoring during labour: a systematic review and thematic analysis. BMC Pregnancy Childbirth. 2012;12(1):166.

22. Whittemore R, Knafl K. The integrative review: updated methodology. J Adv Nurs. 2005;52(5):546-553.

23. Evans D. Integrative reviews of quantitative and qualitative research. In: Webb C, Roe B, editors. Reviewing Research Evidence for Nursing Practice. Oxford, UK: Blackwell Publishing; 2007:135-156.

24. Pluye P, Robert E, Cargo M, et al. Proposal: A Mixed Methods Appraisal Tool for Systematic Mixed Studies Reviews. Montréal: McGill University; 2011.

25. Pace R, Pluye P, Bartlett G, et al. Testing the reliability and efficiency of the pilot mixed methods appraisal tool (MMAT) for systematic mixed studies review. Int J Nurs Stud. 2012;49(1):47-53.

26. Quirk A, Lelliott P, Seale C. Service users' strategies for managing risk in the volatile environment of an acute psychiatric ward. Soc Sci Med. 2004;59(12):2573-2583.

27. Bensley L, Nelson N, Kaufman J, Silverstein B, Shields JW. Patient and staff views of factors influencing assaults on psychiatric hospital employees. Issues Ment Health Nurs. 1995;16(5):433-446.

28. Benson A, Secker J, Balfe E, Lipsedge M, Robinson S, Walker J. Discourses of blame: accounting for aggression and violence on an acute mental health inpatient unit. Soc Sci Med. 2003;57(5):917-926.

29. Bonner G, Lowe T, Rawcliffe D, Wellman N. Trauma for all: a pilot study of the subjective experience of physical restraint for mental health inpatients and staff in the UK. J Psychiatr Ment Health Nurs. 2002; 9(4):465-473. 
30. Ilkiw-Lavalle O, Grenyer BF. Differences between patient and staff perceptions of aggression in mental health units. Psychiatr Serv. 2003; 54(3):389-393.

31. Johnson B, Martin ML, Guha M, Montgomery P. The experience of thought-disordered individuals preceding an aggressive incident. J Psychiatr Ment Health Nurs. 1997;4:213-220.

32. Johnson ME, Delaney KR. Keeping the unit safe: the anatomy of escalation. J Am Psychiatr Nurses Assoc. 2007;13(1):42-52.

33. Omerov M, Edman G, Wistedt B. Violence and threats of violence within psychiatric care - a comparison of staff and patient experience of the same incident. Nord J Psychiatry. 2004;58(5):363-369.

34. Carlsson G, Dahlberg K, Ekebergh M, Dahlberg H. Patients longing for authentic personal care: a phenomenological study of violent encounters in psychiatric settings. Issues Ment Health Nurs. 2006;27:287-305.

35. Cullberg J. Psykoser - Et integrert perspektiv. 2nd ed. Stockholm: Bokforlaget Natur og Kultur; 2005.

36. Bjørkly S. High-risk factors for violence. In: Hodgins, editor. Violence among the Mentally Ill - Effective Treatment and Management Strategies. Netherland: Kluwer Academic Publishers; 2000:237-250.

37. Miller SD, Hubble MA, Duncan BL. The Heart and Soul of Change: What Works in Therapy. Washington, DC: American Psychology Association; 1999.

38. Hewitt J, Coffey M. Therapeutic working relationships with people with schizophrenia: literature review. J Adv Nurs. 2005;52(5):561-570.

39. Vatne S, Fagermoen M. To correct and to acknowledge: two simultaneous and conflicting perspectives of limit - setting in mental health nursing. J Psychiatr Ment Health Nurs. 2007;14(1):41-48.

40. Cleary M, Hunt GE, Horsfall J, Deacon M. Nurse-patient interaction in acute adult inpatient mental health units: a review and synthesis of qualitative studies. Issues Ment Health Nurs. 2012;33(2):66-79.

41. Lendemeijer B, Shortridge-Baggett L. The use of seclusion in psychiatry: a literature review. Sch Inq Nurs Pract. 1996;11(4):299-315. [discussion 220-317].
42. Whittington R, Richter D. From the individual to the interpersonal: environment and interaction in the escalation of violence in mental health settings. In: Richter D, Whittington R, editors. Violence in Mental Health Settings - Causes, Consequences, Management. New York: Springer; 2006:47-68.

43. Papadopoulos C, Ross J, Stewart D, Dack C, James K, Bowers L. The antecedents of violence and aggression within psychiatric in - patient settings. Acta Psychiatr Scand. 2012;125(6):425-439.

44. Coleman PT, Kugler KG, Goldman JS. The Priviledge of Humiliation: The Effects of Social Roles an Normes on immediate and Prolonged Aggression in Conflict. Florida: International Association for Conflict Management (IACM); 2007.

45. Askheim OP, Starrin B. Empowerment i Teori og Praksis. Oslo: Gyldendal Norsk Forlag; 2007.

46. Oeye C, Bjelland AK, Skorpen A, Anderssen N. User participation when using milieu therapy in a psychiatric hospital in Norway: a mission impossible? Nurs Inq. 2009;16(4):287-296.

47. Urheim R, Rypdal K, Palmstierna T, Mykletun A. Patient autonomy versus risk management: a case study of change in a high security forensic psychiatric ward. Int J Forensic Ment Health. 2011;10(1):41-51.

48. Garrod L, Khwaja M, Cumming I. Post-incident management. In: Khwaja M, Beer D, editors. Prevention and Management of Violence: Guidance for Mental Health Care Professionals. London: The Royal College of Psychiatrists; 2013:63-66.

49. Bonner G, Wellmann N. Postincident review of aggression and violence in mental health settings. J Psychosoc Nurs Ment Health Serv. 2010; 48(7):35-40.

50. Fluttert FA, Van Meijel B, Nijman H, Bjørkly S, Grypdonck M. Preventing aggressive incidents and seclusions in forensic care by means of the 'Early Recognition Method'. J Clin Nurs. 2010;19(11-12): 1529-1537.
Journal of Multidisciplinary Healthcare

\section{Publish your work in this journal}

The Journal of Multidisciplinary Healthcare is an international, peerreviewed open-access journal that aims to represent and publish research in healthcare areas delivered by practitioners of different disciplines. This includes studies and reviews conducted by multidisciplinary teams as well as research which evaluates the results or conduct of such teams or

\section{Dovepress}

healthcare processes in general. The journal covers a wide range of areas and welcomes submissions from practitioners at all levels, from all over the world. The manuscript management system is completely online and includes a very quick and fair peer-review system. Visit http://www.dovepress.com/testimonials.php to read real quotes from published authors. 